\section{Topical Anesthesia with Ropivacaine and Proparacaine for Intravitreal Injection Procedures}

\author{
Nektarios E Klados ${ }^{1,3 *}$, Dimitrios T Kourkoutas ${ }^{1}$, Zoi G \\ Kapsala $^{3}$, Eirini-Areti D Giannopoulou ${ }^{3}$ and Miltiadis K \\ Tsilimbaris $^{2,3}$
}

${ }^{1}$ Department of Ophthalmology, 401 General Army Hospital of Athens, Athens, Greece

${ }^{2}$ Department of Ophthalmology, Faculty of Medicine, University Hospital of Heraklion, Crete, Heraklion, Greece

${ }^{3}$ Institute of Vision \& Optics, Faculty of Medicine, School of Health Sciences, University of Crete, Crete, Greece

\begin{abstract}
Purpose: To evaluate the efficacy of the combination of ropivacaine $0.2 \%$ plus proparacaine $0.5 \%$ for topical anesthesia in office intravitreal injection procedures and to compare it with the anesthetic efficacy of proparacaine $0.5 \%$ eye drops alone.

Methods: Prospective study. We compared two different Anesthetic Methods (AM); proparacaine $0.5 \%+$ ropivacaine $0.2 \%$ (AM 1) and proparacaine $0.5 \%$ alone (AM 2). One hundred and fifty five sequential patients undergoing intravitreal injections were randomized into 1 of 3 groups: Group 1 was a crossover group in which patients undertook bilateral same-day intravitreal therapy with different anesthetic method for each eye and compared pain sensation between the two eyes. Group $2 \& 3$ undertook unilateral therapy, received topical anesthesia with anesthetic method $1 \& 2$, respectively, and were asked to grade pain sensation. A 0 to 10 pain scale representing no pain (0) to severe pain (10) was used.

Results: In the first group the 38 patients (76 eyes) were given different anesthesia for each eye before same-day bilateral intravitreal injection with 30 -gauge needle. The average pain score was $2.61 \pm 2.021$ for AM 1 and $3.26 \pm 2.522$ for AM 2. The difference in pain score between the two anesthetic methods was statistically significant $(P=0.039)$. For group 2 the average pain score was $2.38 \pm 1.795$ while for group 3 it was $2.66 \pm 2.014(P=0.426)$.
\end{abstract}

*Corresponding author: Nektarios E Klados, Department of Ophthalmology, 401 General Army Hospital of Athens, 138 Mesogion \& Katehaki Ave, 11525 Athens, Greece, Tel: +30 2107494824; Fax: +30 2107494095; E-mail: neklados@gmail.com

Citation: Klados NE, Kourkoutas DT, Kapsala ZG, Giannopoulou ED, Tsilimbaris MK (2015) Topical Anesthesia with Ropivacaine and Proparacaine for Intravitreal Injection Procedures. J Ophthalmic Clin Res 2: 014.

Received: September 03, 2015; Accepted: October 20, 2015; Published: November 03, 2015
Conclusion: The combination of proparacaine $0.5 \%$-as short acting anesthetic-with ropivacaine $0.2 \%$-as long acting anesthetic-seems to be an effective anesthetic option which provides patients with high rates of pain control and overall satisfaction during the office based intravitreal injection procedures.

Keywords: Intravitreal injection; Proparacaine; Ropivacaine

\section{Introduction}

Intravitreal injection represents an efficient method to achieve high intraocular levels of drugs into the posterior segment of the eye. For many years, this method has been used for the intravitreal administration of antiviral, antibiotic or antifungal drugs, steroids and gas. Since the mid of the previous decade, the advent of anti-VEGF injections as an effective therapy for neovascular Age-related Macular Degeneration (AMD) and for macular oedema due to diabetes or retinal vein occlusion, intravitreal injections have become one of the most common intraocular procedure performed worldwide. The repeatability of the anti-VEGF injections for a sufficient vision-saving treatment and the fact that the patient is required to return on a regular basis for subsequent injections have turned patient comfort into a crucial parameter during the overall procedure.

Previous studies suggest that among the anesthetic techniques (including subconjunctival and peribulbar injection), the topical administration of anesthetics is the most comfortably applied, cost-effective, and easiest way to achieve anesthesia [1-4]. Moreover, recent studies have compared the effectiveness of different topical anesthetic drugs without yielding a consensus concerning the superiority of any of the agents tested [2,5].

Ropivacaine is a monoamide local anesthetic that has been successfully used for topical anesthesia during several ocular operations but, to our knowledge, it has not been tried yet as a topical anesthetic for intravitreal injection procedures [6].

In this view, we designed a study to evaluate the effectiveness of ropivacaine in combination with proparacaine as topical anesthesia for intravitreal injections and to compare it with proparacaine alone.

\section{Patients and Methods}

This prospective study was conducted at the retina service, University Hospital of Heraklion, Crete, after it was approved by our hospital's institutional review board. All participants gave written informed consent to participate in the study. All patients had received at least one previous intravitreal injection for any etiology. Exclusion criteria included any known allergy or sensitivity to the study medications, known diabetic peripheral neuropathy as well as patients with previous ocular surgery (other than cataract surgery). The Anesthetic Methods (AM) that were compared were: AM 1, that included the instillation of one drop of proparacaine $0.5 \%$ (Alcaine Ey. Dro.Sol. 0.5\%; Alcon Laboratories A.E.B.E.) before application of $10 \%$ povidone-iodine antisepsis (Betadine Cut. Sol. 10\%; Lavipharm A.E.) to the eyelids and periorbital area and the placement of a sterile solid lid speculum followed by one drop of ropivacaine $0.2 \%$ 
[Naropeine Inj. Sol. 0.2\% (2 mg/mL), AstraZeneca Hellas] for three consecutive times in a three-minute period; and AM 2, that included the instillation of one drop of proparacaine $0.5 \%$ (Alcaine Ey. Dro. Sol. 0.5\%; Alcon Laboratories A.E.B.E.) before application of $10 \%$ povidone-iodine antisepsis (Betadine Cut. Sol. 10\%; Lavipharm A.E.) to the eyelids and periorbital area and the placement of a sterile solid lid speculum followed by one drop of proparacaine $0.5 \%$ (Alcaine Ey. Dro. Sol. 0.5\%; Alcon Laboratories A.E.B.E.) for three consecutive times in a three-minute period. Additionally, povidone-iodine 5\% was instilled to the conjunctiva over the injection site at the intervals of the anesthetic's instillations in both anesthetic methods. All intravitreal injections were performed by the same practitioner using sterile gloves 2 minutes after the completion of anesthesia. Sterile calipers were used to measure the injection site $3.5 \mathrm{~mm}$ (pseudophakic) or $4.0 \mathrm{~mm}$ (phakic) posterior to the superior temporal limbus. A 30-gauge needle (Becton Dickinson \& Co., Franklin Lakes, NJ, Precision glide \#305106) was used to inject 0.05 $\mathrm{mL}$ of either ranibizumab (Lucentis, Novartis Europharm Ltd) or bevacizumab (Avastin, Roche registration Ltd). A drop of tobramycin $0.3 \%$ was then applied topically over the injection site and also used to rinse excess Betadine from the eye surface. The preparation of eye drops in both methods was performed in a daily manner.

Three groups of patients were enrolled. Study patients scheduled for same-day bilateral therapy were included in the $1^{\text {st }}$ group while study patients scheduled for unilateral therapy were randomized to $2^{\text {nd }}$ and $3^{\text {rd }}$ treatment groups. Group 1 was a crossover group in which patients undertook bilateral same-day intravitreal therapy with different anesthetic method for each eye. Both anesthetic method and therapy were randomized to each eye. Completing the bilateral process, the patients had to grade pain sensation in each eye separately. Group 2 undertook unilateral therapy, received topical anesthesia with anesthetic method 1 and were asked to grade pain sensation. Group 3 undertook unilateral therapy, received topical anesthesia with anesthetic method 2 and were asked to grade pain sensation (Tables 1 and 2).

\begin{tabular}{|c|c|c|c|}
\hline & AM 1 & AM 2 & \multirow{2}{*}{ P value } \\
\hline Age & \multicolumn{2}{|c|}{ Mean=70.26 } & \\
\hline Gender & \multicolumn{2}{|c|}{$\begin{array}{r}\text { Male=18 } \\
\text { Female=20 }\end{array}$} \\
\hline VA Change & 0.02189 & -0.00245 & \multirow{2}{*}{$\mathrm{P}=0.242$} \\
\hline Number of injections & 10.18 & 9.18 & $\mathrm{P}=0.359$ \\
\hline
\end{tabular}

Table 1: Group 1 (Crossover) demographic characteristics of patients and clinical history.

\begin{tabular}{|c|c|c|c|}
\hline & AM 1 (Group 2) & AM 2 (Group 3) & P value \\
\hline Age & Mean=72.64 & Mean=71.32 & $\mathrm{P}=0.420$ \\
\hline \multirow{2}{*}{ Gender } & Male=30 & Male=33 & \multirow{2}{*}{$\mathrm{P}=0.712$} \\
\cline { 2 - 3 } & Female=28 & Female=26 & \\
\hline VA Change & -0.048284 & 0.020610 & $\mathrm{P}=0.018$ \\
\hline Number of injections & 9.66 & 11.05 & $\mathrm{P}=0.499$ \\
\hline
\end{tabular}

Table 2: Group 2 \& 3; Demographic characteristics of patients and clinical history.

Five to ten minutes after the end of the process, a trained para-ophthalmic technician performed the pain grading procedure using a 10-degree Visual Analog Scale (VAS) for pain score estimation [1]. The technician explained to the patient that point 0 of the scale represented "no pain at all" while point 10 represented "the most intense pain one could ever feel". The patient was asked about the intensity of pain during the intravitreal injection.

\section{Results}

All the procedures were successfully performed with good patient cooperation. No complication associated with the anesthetic methods or the intravitreal injections occurred in the groups.

In the first group the 38 patients (76 eyes) were given different anesthesia for each eye before same-day bilateral intravitreal injection with 30 -gauge needle. The mean age was 70 years, with a range from 32 years to 88 years. Most of the patients (24/38) took therapy for diabetic macular oedema, 13 of 38 took therapy for a neovascular Age-Related Macular Degeneration (ARMD) while one of them took therapy for bilateral macular oedema caused by retinal vein occlusion. Ranibizumab was injected into 38 eyes of 38 patients and bevacizumab was injected into the other 38 eyes of these patients. There was no significant difference in visual acuity change $(\mathrm{P}=0.242)$ between the eyes. Moreover, all the eyes had at least 1 previous intravitreal injection, with a range of 1 to $27($ mean=10.18) for eyes received AM 1 and 1 to 22 (mean $=9.18$ ) for eyes received AM $2(\mathrm{P}=0.359)$. The average pain score was $2.61 \pm 2.021$ ( $\mathrm{SD}=1.547)$ for $\mathrm{AM} 1$ and $3.26 \pm 2.522(\mathrm{SD}=1.598)$ for $\mathrm{AM} 2$. The difference in pain score between the two anesthetic methods was statistically significant $(\mathrm{P}=0.039)$ (Table3).

\begin{tabular}{|c|c|c|c|c|}
\hline & Group & AM 1 & AM 2 & P value \\
\hline \multirow{3}{*}{ Pain score } & 1 & 2.61 & 3.26 & $\mathrm{P}=0.039$ \\
\cline { 2 - 5 } & 2 & 2.38 & & \multirow{2}{*}{$\mathrm{P}=0.426$} \\
\cline { 2 - 4 } & 3 & & 2.66 & \\
\hline
\end{tabular}

Table 3: Comparison of pain sensation after Proparacaine $0.5 \%+$ Ropivacaine $0.2 \%$ (AM 1) versus Proparacaine $0.5 \%$ (AM 2) in the study.

In the second group, 58 patients (58 eyes), were given anesthesia with AM 1 before unilateral intravitreal injection with 30-gauge needle. The mean age was 73 years, with a range from 48 years to 86 years. All the patients had at least 1 previous intravitreal injection, with a range of 1 to 49 (mean=9.66). Most of the patients $(29 / 58)$ took therapy for neovascular Age-Related Macular Degeneration (ARMD), 22 of 58 took therapy for diabetic macular oedema while seven of them took therapy for macular oedema caused by retinal vein occlusion. Ranibizumab was injected into 42 eyes and bevacizumab was injected into 16 eyes. In the third group, 59 patients (59 eyes), were given anesthesia with AM 2 before unilateral intravitreal injection with 30 -gauge needle. The mean age was 71 years, with a range from 34 years to 88 years. All the patients had at least 1 previous intravitreal injection, with a range of 1 to $50($ mean=11.05). Most of the patients (27/59) took therapy for diabetic macular oedema, 24 of 59 took therapy for neovascular Age-Related Macular Degeneration (ARMD) while eight of them took therapy for macular oedema caused by retinal vein occlusion. Ranibizumab was injected into 35 eyes and bevacizumab was injected into 24 eyes. There was no significant difference in gender $(\mathrm{P}=0.648)$, injected drug $(\mathrm{P}=0.269)$ and disease $(\mathrm{P}=0.857)$ between these two groups. However, there was a significant difference in visual acuity change $(\mathrm{P}=0.018)$. The average pain score was $2.38 \pm 1.795(\mathrm{SD}=1.342)$ for group 2 and $2.66 \pm 2.014(\mathrm{SD}=1.734)$ for group $3(\mathrm{P}=0.426)$ (Table 3$)$.

\section{Discussion}

Intravitreal injections represent an excellent, direct-route method to deliver high concentrations of pharmacologic agents into the 
posterior segment of the eye. However, this method is definitely painful and stressful for the patient. Moreover, patients are required to return on a regular basis for subsequent injections as frequently as every month and these may continue for years. It is clear that the anesthetic option for a sufficient patient comfort is an extremely important factor for a successful overall therapy.

Previous studies suggest that among the anesthetic techniques (including SC and peribulbar injection), topical administration could be considered the gold standard anesthetic method for the office-based intravitreal injection procedure, as it is the most comfortably applied, cost-effective, and easiest to administer [1-4]. Additionally, in a recently published study, Sanabria et al., concluded that topical tetracaine and lidocaine provide similar anesthesia before intravitreal injections and that topical diclofenac does not seem to reduce pain scores after them [5]. In agreement with this conclusion, Yau et al., showed that there was no difference in patient pain experience between the 3 (tetracaine, lidocaine and cocaine) anesthetic options tested [7]. Similarly, Blaha et al., compared lidocaine pledget, topical proparacaine, subconjunctival lidocaine and topical tetracaine without obtaining any significant difference in patient pain experience [8].

Ropivacaine is the S-enantiomer of 1-propyl-2', 6'-pipecoloxylidide. It is a new, long-acting local anesthetic of the amide type with minimal cardiological and neurological toxic events. Several studies have been done to reveal the scope of ropivacaine for use in various diseases and conditions [6]. In ophthalmology, topical anesthesia with ropivacaine is safe and effective in pterygium surgery. Caccavale et al., showed that the long-lasting anesthesia with this agent permitted performing the surgical procedures with autograft conjunctival graft and fibrin glue to attach the flap with low pain perceived by the patients, low surgical invasivity and short duration of surgery [9]. Moreover, Martini et al., referred that topical ropivacaine performed at least as well as topical lidocaine in efficacy and safety in cataract surgery. It provided sufficient and long-lasting analgesia without the need for supplemental intracameral anesthesia in most cases [10]. In a relative study, Borazan et al., concluded that topical anesthesia with ropivacaine was safe, feasible and more effective than lidocaine in cataract surgery [11].

In our study, we tried to evaluate the effectiveness of the combination of ropivacaine $0.2 \%$ and proparacaine $0.5 \%$ (AM 1), as a topical anesthetic option, for office-based intravitreal injection procedure and to compare it with proparacaine $0.5 \%$ alone (AM 2). We were able to show that both anesthetic methods in an over-all duration of 5 minutes provided satisfactory patient comfort for administration of intravitreal injection. In the $1^{\text {st }}$ group of patients with bilateral same-day intravitreal injection, the combined Anesthetic Method (AM 1) applied in one eye offered a clinically significant advantage in pain compared to proparacaine $0.5 \%$ alone applied in the other eye. Both ropivacaine and proparacaine cause reversible inhibition of sodium ion influx, and thereby block impulse conduction in nerve fibres. Moreover, ropivacaine has the property to potentiate this action with dose-dependent inhibition of potassium channels and has a long acting anesthetic outcome [12].

The anesthetic methods (AM 1 \& AM 2) were also used in two different groups $(2 \& 3)$ of the patients who undertook unilateral intravitreal therapy. Our results suggested that patients of $2^{\text {nd }}$ group had a better pain relief (2.38) than patients of $3^{\text {rd }}$ one (2.66) although this difference was not statistically significant. Interestingly, the vision change was different between these groups. In $2^{\text {nd }}$ group the vision was reduced whereas, in $3^{\text {rd }}$ group, the vision was improved and this result may explain the non-statistical significant difference between the anesthetic methods in these two groups. In a recent study, patients whose vision had improved from their previous injection reported a significantly lower average pain score than those who did not show improvement [13]. Perhaps the mental satisfaction with the knowledge that the treatment was working helped patients overcome the physical pain that they were likely to experience.

Before commencing our study, the only published data on topical ocular surface anesthesia with ropivacaine were cataract and pterygium extraction operations [9-11]. We wished to determine whether ropivacaine as the sole anesthetic could provide satisfactory ocular surface anesthesia for intraocular injection procedure. However, before commencing the study, our impression with ropivacaine $0.02 \%$ was that it provoked patient's itching and burning sensation topically. Hence, we opted for a combination of proparacaine $0.05 \%$ with ropivacaine $0.02 \%$, as ropivacaine is not currently available in ophthalmic solution. It should be interesting to try out an ophthalmic solution of ropivacaine in the future, as the sole anesthetic for intravitreal injections, avoiding the relative topical adverse effects.

To date, our study validates the reports that topical anesthesia can be considered as the gold standard anesthetic method for intravitreal injections. Moreover, we conclude that the combination of proparacaine $0.5 \%$-as short acting anesthetic-with ropivacaine $0.2 \%$-as long acting anesthetic-seems to be an effective anesthetic option which provides patients with high rates of pain control and overall satisfaction during the office based intravitreal injection procedure and may be superior to proparacaine $0.5 \%$ alone. A future production of ropivacaine's ophthalmic solution should be considerably useful and could provide another efficient way of topical anesthesia for several ocular operations.

\section{References}

1. Cintra LP, Lucena LR, Da Silva JA, Costa RA, Scott IU, et al. (2009) Comparative study of analgesic effectiveness using three different anesthetic techniques for intravitreal injection of bevacizumab. Opthalmic Surg Lasers Imaging 40: 13-18.

2. Kaderli B, Avci R (2006) Comparison of topical and subconjunctival anesthesia in intravitreal injection administrations. Eur J Ophthalmol 16: 718-721.

3. Kozak I, Cheng L, Freeman WR (2005) Lidocaine gel anesthesia for intravitreal drug administration. Retina 25: 994-948.

4. Friedman SM, Margo CE (2006) Topical gel vs subconjunctival lidocaine for intravitreous injection: a randomized clinical trial. Am J Ophthalmol 142: 887888.

5. Sanabria MR, Montero JA, Losada MV, Fernández-Muñoz M, Galindo A, et al. (2013) Ocular pain after intravitreal injection. Curr Eye Res 38: 278-282.

6. Chandrasekhar N, Jayasree T, Vinay M, Dixit R, Manohar VS, et al. (2011) Action of ropivacaine as a surface anaesthetic on the cornea of rabbits. International Journal of Pharmacology 7: 273-277.

7. Yau GL, Jackman CS, Hooper PL, Sheidow TG (2011) Intravitreal injection anesthesia--comparison of different topical agents: a prospective randomized controlled trial. Am J Ophthalmol 151: 333-337.

8. Blaha GR, Tilton EP, Barouch FC, Marx JL (2011) Randomized trial of anesthetic methods for intravitreal injections. Retina 31: 535-539.

9. Caccavale A, Romanazzi F, Imparato M, Negri A, Porta A, et al. (2010) Ropivacaine for topical anesthesia in pterygium surgery with fibrin glue for conjunctival autograft. Cornea 29: 375-376. 
Citation: Klados NE, Kourkoutas DT, Kapsala ZG, Giannopoulou ED, Tsilimbaris MK (2015) Topical Anesthesia with Ropivacaine and Proparacaine for Intravitreal Injection Procedures. J Ophthalmic Clin Res 2: 014.

- Page 4 of $4 \cdot$

10. Martini E, Cavallini GM, Campi L, Lugli N, Neri G, et al. (2002) Lidocaine versus ropivacaine for topical anesthesia in cataract surgery. $\mathrm{J}$ Cataract Refract Surg 28: 1018-1022.

11. Borazan M, Karalezli A, Akova YA, Algan C, Oto S (2008) Comparative clinical trial of topical anaesthetic agents for cataract surgery with phacoemulsification: lidocaine $2 \%$ drops, levobupivacaine $0.75 \%$ drops, and ropivacaine $1 \%$ drops. Eye (Lond) 22: 425-429.
12. Kindler CH, Paul M, Zou H, Liu C, Winegar BD, et al. (2003) Amide local anaesthetics potently inhibit the human tandem pore domain background $\mathrm{K}^{+}$ channel TASK-2 (KCNK5). J Pharmacol Exp Ther 306: 84-92.

13. Rifkin L, Schaal S (2012) Factors affecting patients' pain intensity during in office intravitreal injection procedure. Retina 32: 696-700. 